\section{Um exame dos fluxos financeiros do Ministério da Saúde em pesquisa e desenvolvimento (2003-2005), segundo a Agenda Nacional de Prioridades de Pesquisa em Saúde}

\author{
An analysis of financial flows from the Brazilian \\ Ministry of Health for research and development \\ in 2003-2005 according to the National Agenda \\ for Health Research Priorities
}

Rondineli Mendes da Silva 1,2 Rosangela Caetano 1

\section{Introdução}

This study mapped the application of financing in research and development in health $(R \& D / H)$ by the Brazilian Ministry of Health in 2003-2005, according to the National Agenda for Health Research Priorities, created in 2004. The analysis was based on data from a study aimed primarily at measuring these investment flows during the same period. The calculations included only direct financing with actual outlays in research, including payroll expenditures. The studies were categorized according to the 24 sub-agendas of the national priority agenda by two independent researchers, and disagreements were resolved by consensus. Research and development expenditures in health totaled 409.7 million reais, concentrated mainly in the following sub-agendas: transmissible diseases, the health industry complex, clinical research, pharmaceutical care, and non-communicable diseases (79\% of the total). All 24 sub-agendas received some financing during the period. The study established a baseline for subsequent evaluations of this financing instrument's inductive capacity and the relationship between R\&D/H investments and the population's health needs.

Financial Resources in Health; Health Priority Agenda; Health Research Policy
Excetuando-se a pesquisa militar, o setor saúde é onde se despende maior volume de recursos financeiros em pesquisa em termos mundiais 1 . Segundo dados do Global Forum for Health Research $^{2}$, os gastos mundiais em pesquisa e desenvolvimento em saúde (P\&D/S), em 2005, foram de US\$ 160,3 bilhões, representando aumento superior a 50\% quando comparado a US\$ 105,9 bilhões investidos em 2001.

Apesar desse montante representativo, são observadas situações injustas na sua divisão quando comparados sob a lógica das necessidades de saúde, com uma dissociação entre a carga de doenças das populações mais desfavorecidas e a concentração do investimento global em $\mathrm{P} \& \mathrm{D} / \mathrm{S}$ nos problemas do mundo industrializado. Essa situação de deficiência no processo de estabelecimento e de execução de um conjunto de prioridades em pesquisa tem conduzido a uma situação na qual menos de $10 \%$ dos recursos financeiros, públicos e privados, destinados à pesquisa são devotados a $90 \%$ dos problemas de saúde que afetam a população mundial (gap 10/90) 3 .

No Brasil, os recursos destinados a $\mathrm{P} \& \mathrm{D} / \mathrm{S}$ são ainda bastante escassos. Ademais, as atividades de pesquisa em saúde tradicionalmente eram (e sob diversos aspectos, ainda são) desenvolvidas sem uma articulação maior com a Política Nacional de Saúde 4, devendo, portanto, ser 
formuladas no âmbito do SUS. Tal conexão já se encontrava expressa na Constituição Federal de 1988 que, dentre as competências do SUS, lista o incremento do desenvolvimento científico e tecnológico em sua área de atuação.

Um dos caminhos concebidos para a correção destas deficiências é o estabelecimento de agenda de prioridades de pesquisa, discutido nas Conferências Nacionais de Ciência e Tecnologia em Saúde de 1994 e 2004. Esta modalidade de ação tem sido apresentada por diversas instâncias internacionais e foi recentemente adotada pelo Ministério da Saúde, como uma de suas diretrizes atuais, no contexto mais amplo das Políticas Nacionais de Saúde e de Ciência e Tecnologia 5,6 .

Esse mecanismo de agenda permite aumentar a capacidade indutora do sistema de fomento científico-tecnológico com base em uma escolha racional de prioridades, minimizando uma das características históricas desse sistema, sua baixa seletividade, e contribuindo para reduzir as iniquidades em saúde e fortalecer o setor científico-tecnológico 4.

Em 2004, o Ministério da Saúde estabeleceu a Política Nacional de Ciência e Tecnologia e Inovação em Saúde (PNCTI/S) 7, sendo uma de suas principais estratégias a construção da Agenda Nacional de Prioridades de Pesquisa em Saúde (ANPPS), aprovada em 2004. Essa agenda possui, dentre seus pressupostos, os de respeitar as necessidades nacionais e regionais, e aumentar a indução seletiva na produção de conhecimentos e bens materiais e processuais nas áreas prioritárias para o desenvolvimento das políticas sociais 8 .

A agenda está constituída por 24 subagendas, subdividida em diversos níveis: (1) saúde dos povos indígenas; (2) saúde mental; (3) violência, acidentes e trauma; (4) saúde da população negra; (5) doenças não-transmissíveis; (6) saúde do idoso; (7) saúde da criança e do adolescente; (8) saúde da mulher; (9) saúde dos portadores de necessidades especiais; (10) alimentação e nutrição; (11) bioética e ética na pesquisa; (12) pesquisa clínica; (13) complexo produtivo da saúde; (14) avaliação de tecnologias e economia da saúde; (15) epidemiologia; (16) demografia e saúde; (17) saúde bucal; (18) promoção da saúde; (19) doenças transmissíveis; (20) comunicação e informação em saúde; (21) gestão do trabalho e educação em saúde; (22) sistemas e políticas de saúde; (23) saúde, ambiente, trabalho e biossegurança e (24) assistência farmacêutica 8 .

Desde a 12a Conferência Nacional de Saúde, em 2003, se considerava que essa agenda deveria ser levada em consideração pelas agências de fomento científico e tecnológico, constituindo-se em um dos critérios para aprovação de projetos, tendo em vista sua relevância para os problemas de saúde pública 9 . Ao longo deste ano, iniciaramse os primeiros movimentos para sua construção, através da elaboração de documentos para subsidiar as discussões da seleção das áreas prioritárias a serem consideradas e da organização de seminário que reuniu, em novembro de 2003, 408 pesquisadores, gestores e profissionais de saúde, que definiram as linhas prioritárias 6 . O texto da ANPPS foi parcialmente aprovado na $2 \underline{a}$ Conferência Nacional de C\&T\&I/S ocorrida em 25-28 de julho de 2004 e, posteriormente, referendado pela 151a Reunião Ordinária do Conselho Nacional de Saúde, em 17 de fevereiro de 2005 .

Em termos de investimentos, já no ano de construção da ANPPS existiram alguns dispêndios do Ministério da Saúde no financiamento de pesquisas relacionadas a temas prementes da política de saúde 10. Entretanto, sua utilização como eixo orientador em editais de financiamento diretos ou em cooperação técnica com agências de fomento do Ministério de Ciência e Tecnologia, só iniciou-se formalmente partir de 20056 .

O triênio 2003-2005 corresponde, pois, ao período de transição entre investimentos dispersos e o início daqueles mais seletivamente orientados, onde talvez já comece a ocorrer, mesmo que timidamente, a influência indutora da ANPPS. Como toda transição, estas mudanças correspondem a um processo, mais do que a alterações bruscas. Estudar esse período pode ajudar no entendimento de como esse processo foi se expressando no perfil de investimentos e servir a comparações posteriores em séries históricas dos fluxos financeiros do Ministério da Saúde na área de $\mathrm{P} \& \mathrm{D} / \mathrm{S}$ que venham a ser construídas.

Este trabalho objetivou mapear a distribuição dos dispêndios do Ministério da Saúde em P\&D/S no período de 2003-2005 segundo os itens componentes da ANPPS estabelecida em 2004.

\section{Metodologia}

Este trabalho exploratório-descritivo foi estruturado com base na pesquisa Fluxos de Recursos Financeiros para a Pesquisa e Desenvolvimento em Saúde no Brasil 2003-2005 11, realizado por pesquisadores do Instituto de Medicina Social da Universidade do Estado do Rio de Janeiro.

O estudo citado quantificou os investimentos realizados em $\mathrm{P} \& \mathrm{D} / \mathrm{S}$ por organizações nacionais públicas e privadas e por organismos internacionais, usando como critério central para a definição de P\&D/S o de setor de atividade /aplicação na área da saúde, em vez de área de conheci- 
mento em saúde. Desse modo, P\&D/S nesse estudo ampliava-se para outras ciências/áreas de conhecimento (agrárias, sociológicas, humanas etc.) e incluiu financiamentos de pesquisas que não se relacionavam diretamente com áreas do conhecimento convencionalmente ligadas ao setor, mas que apontavam novas aplicações para melhoria da saúde de grupos e de indivíduos.

A pesquisa de base não contemplava o foco do presente trabalho, qual seja, a avaliação da distribuição dos dispêndios segundo a ANPPS, e incluía os salários de pesquisadores de fundações e institutos de pesquisa vinculados ao Ministério da Saúde, impossíveis de serem decompostos segundo os componentes da agenda, como dispêndios em pesquisa. No estudo em tela, apenas os investimentos diretos em $\mathrm{P} \& \mathrm{D} / \mathrm{S}$ do Ministério da Saúde foram analisados. A opção por recortar pelos gastos deste Ministério considerou: (1) seu papel central na formulação e gestão da política de ciência e tecnologia (C\&T) no país; e, (2) o pressuposto de que a capacidade de induzir os investimentos em $\mathrm{P} \& \mathrm{D} / \mathrm{S}$ pela ANPPS tenha se iniciado exatamente pelas pesquisas financiadas por este ente governamental.

Foram considerados apenas investimentos em $\mathrm{P} \& \mathrm{D} / \mathrm{S}$ aplicados pelas administrações diretas (secretarias da estrutura do Ministério da Saúde, Fundo Nacional de Saúde) e indiretas (Fundação Oswaldo Cruz - Fiocruz; Fundação Nacional de Saúde - FUNASA; Agência Nacional de Vigilância Sanitária - ANVISA; e Agência Nacional de Saúde Suplementar - ANSS) do Ministério da Saúde.

A busca de informações foi feita a partir das instituições fontes de financiamento, agentes financiadores primários de pesquisa em saúde, independentemente da existência de intermediários até o usuário final dos recursos. Essa opção se fundamentou: (1) nas características do sistema nacional de C\&T e inovação, basicamente estruturado por agências de fomento públicas federais e estaduais e órgãos setoriais que fomentam pesquisas voltadas para suas políticas; e (2) porque os usuários de recursos de $\mathrm{P} \& \mathrm{D} / \mathrm{S}$, executores finais das pesquisas, têm grande dispersão e, em geral, não possuem sistema contábil que permita identificar a origem dos recursos recebidos para financiamento de pesquisas.

A investigação obedeceu a duas alternativas complementares: (1) levantamento e análise de dados secundários disponíveis nos bancos de dados, relatórios e outros documentos existentes nas páginas de Internet das instituições intraministério; e (2) solicitação de informações específicas, mediante correspondência dirigida às direções e/ou outros responsáveis previamente identificados, com o envio de instrumentos especialmente desenhados e instrutivos de preenchimento. Foram coletadas informações sobre nome do projeto de pesquisa, instituição recebedora dos recursos, coordenador, data de início e término, e valor financiado nos anos sob estudo. Não foram executadas estratégias de validação das informações obtidas, dada a multiplicidade de instituições usuárias de recursos, já mencionadas.

Todas as informações referentes às pesquisas alimentaram banco de dados especialmente construído pelo Instituto de Comunicação e Informação Científica e Tecnológica em Saúde, da Fiocruz.

Foram adotadas estratégias para evitar dupla contagem dos fomentos realizados. Após a entrada no sistema, verificou-se a existência de duplicidades a partir do título da pesquisa e nome do coordenador, confrontando-se com suas respectivas fontes financiadoras.

Algumas decisões metodológicas merecem destaque. Recursos externos advindos de acordos internacionais com o Ministério da Saúde, como aqueles do Programa Nacional de HIV/AIDS, não foram incluídos como investimentos próprios deste Ministério. Sempre que se observou que uma instituição destinatária funcionava, na realidade, como intermediária no fluxo de recursos, os valores foram atribuídos aos usuários executores das atividades de $\mathrm{P} \& \mathrm{D} / \mathrm{S}$. Quando isso não foi possível, os recursos permaneceram atribuídos ao destinatário identificado no processo de coleta de dados, mesmo havendo a clareza que esses intermediários não eram executores finais das pesquisas. Em situações onde uma mesma pesquisa tinha mais de uma fonte de financiamento - por exemplo, quando existem contrapartidas subnacionais a pesquisas financiadas com recursos do Ministério da Saúde (por exemplo, editais do Programa de Apoio a Núcleos de Excelência - PRONEX) - apenas os recursos oriundos do Ministério da Saúde foram considerados.

A distribuição dos investimentos foi realizada conforme a origem dos recursos no organograma do Ministério da Saúde. Já as instituições destinatárias dos recursos foram agrupadas em: órgãos e fundações do Ministério da Saúde; órgãos e fundações vinculadas de outros ministérios; Fundações de Amparo à Pesquisa (FAP); outros órgãos estaduais e municipais; organizações não-governamentais; conselhos profissionais e associações de especialistas; universidades, institutos de pesquisa e fundações vinculadas; instituições outras do setor privado; e, organismos internacionais. Fez-se ainda uma avaliação de sua distribuição pelas regiões do país.

Os dispêndios do Ministério da Saúde, dispostos segundo sua estrutura organizacional e a categorização institucional dos receptores dos 
recursos, permitiram a construção da respectiva matriz de fluxos dos financiamentos do Ministério da Saúde para os diversos atores envolvidos no processo de $\mathrm{P} \& \mathrm{D} / \mathrm{S}$ no período.

As pesquisas foram categorizadas por natureza (pesquisa básica, aplicada e desenvolvimento experimental) e campo de atividades (ciências naturais; ciências médicas; ciências sociais/economia da saúde; outros campos de P\&D/S com aplicação na área da saúde), seguindo a metodologia desenvolvida pelo Center for Economic Policy Research 12, adaptada para a realidade brasileira 13 .

Por fim, os dispêndios financeiros em $\mathrm{P} \& \mathrm{D} / \mathrm{S}$ foram categorizados segundo as 24 subagendas componentes, até o segundo nível da ANPPS, de modo independente, por dois pesquisadores, com as discordâncias resolvidas por consenso. Todas as pesquisas foram classificadas em até três itens desta Agenda, porque algumas possuíam focos múltiplos, como, por exemplo, estudos voltados a doenças infecciosas em crianças.

O programa Stata 8.0 (Stata Corp., College Station, Estados Unidos) foi utilizado nas análises estatísticas.

\section{Resultados}

$\mathrm{O}$ volume de investimentos diretos em $\mathrm{P} \& \mathrm{D} / \mathrm{S}$ pelo Ministério da Saúde, entre 2003-2005, foi de aproximadamente R \$409,7 milhões (US\$ 153 milhões), com média anual de R\$ 136,6 milhões.

A administração indireta do Ministério da Saúde investiu cerca de R\$ 229 milhões, correspondendo a $55,9 \%$ do total. Destacam-se, como principais fontes de financiamento, a Fiocruz, órgão da Administração Indireta, e a Secretaria de Ciência, Tecnologia e Insumos Estratégicos (SCTIE), pertencente à Administração Direta, que juntas responderam por quase $80 \%$ do total de investimentos aplicados.

A distribuição dos recursos por regiões demonstra a supremacia do Sudeste, comportando $72,5 \%$ do total de investimentos, seguida do Nordeste (12,6\%), enquanto $6 \%, 5,9 \%$ e $2,9 \%$ se referem às regiões Norte, Sul e Centro-oeste, respectivamente. Todas as 27 Unidades Federativas receberem algum recurso de $\mathrm{P} \& \mathrm{D} / \mathrm{S}$ no período, demonstrando que já se faz presente algum grau de distributividade na pesquisa no país, mormente persistam significativas desigualdades. Ressalta-se a relevância do Estado do Rio de Janeiro, com 53,8\% de aplicação de recursos, seguido de São Paulo (14\%) e Bahia (6,4\%). A participação preponderante do Rio de Janeiro se explica, em parte, pela localização neste estado dos maiores Institutos de pesquisas componentes da Fiocruz, que possuem recursos próprios orçamentários de $\mathrm{P} \& \mathrm{D} / \mathrm{S}$.

A distribuição segundo categoria institucional dos receptores dos recursos mostrou que os órgãos e entidades do Ministério da Saúde (incluindo suas fundações) receberam $55,9 \%$ do volume, o que é grandemente explicado também pela presença da Fiocruz. Em seguida, aparecem as Universidades, Institutos de pesquisa e fundações (32\%), com participação aumentada nos últimos anos a partir de iniciativas de financiamento do Departamento de Ciência e Tecnologia da Secretaria de Ciência e Tecnologia e Insumos Estratégicos (DECIT/SCTIE), entre os quais se incluem os editais temáticos, lançados em conjunto com o Conselho Nacional de Desenvolvimento Científico e Tecnológico/Financiadora de Estudos e Projetos (CNPq/FINEP), e aqueles relativos ao PPSUS-Gestão Compartilhada, com o apoio das FAP.

Ressalte-se também que as FAP não são, em si, executoras diretas de pesquisas e sim funcionam como intermediárias nos fluxos de recursos. Assim, sempre que foi possível identificar o destinatário final, sua categoria respondeu pelos recursos recebidos. O volume financeiro que restou atribuído a categoria institucional FAP $(0,16 \%$ do total) representa, pois, projetos onde não se pode identificar o usuário final executor da pesquisa.

Pequenos volumes de recursos foram destinados a pesquisas executadas por órgãos de outros ministérios, como é o caso da Empresa Brasileira de Pesquisa Agropecuária (EMBRAPA) e do Instituto Nacional de Metrologia, Normalização e Qualidade Industrial (INMETRO), pertencentes respectivamente aos Ministérios da Agricultura e do Desenvolvimento, Indústria e Comércio Exterior, bem como a estados e municípios, como por exemplo, investigações destinadas a caracterizar o perfil de sensibilidade aos antibióticos de amostras de enterococos isolados da carne de frango e o diagnostico situacional dos conselhos municipais de saúde do Acre.

A categoria organismos internacionais $(2,4 \%$ do total) corresponde a situações onde houve repasse de recursos para instituições como Organização Pan-Americana da Saúde (OPAS) e Organização das Nações Unidas para a Educação a Ciência e a Cultura (UNESCO), que posteriormente financiaram pesquisas em território nacional, mas onde não foi possível identificar quais os destinatários finais destes investimentos.

Em relação à natureza das pesquisas financiadas, estudos sem informação suficiente que permitisse sua categorização corresponderam a 6,6\% do total ( $\mathrm{R} \$ 27$ milhões). Do restante, $\mathrm{R} \$$ 233,9 milhões foram investidos em pesquisa aplicada $(57,1 \%)$ e R\$ 79,6 milhões em desenvolvi- 
mento experimental (19,4\%), enquanto 3,7\% (R\$ 15 milhões) foram aplicados em pesquisa básica. Investimentos em projetos relacionados à infraestrutura em C\&T/S responderam por $13,2 \%$ dos recursos.

No tocante ao campo de atividade das pesquisas, $6,3 \%$ do total ficaram sem informação; do restante, investimentos em ciências médicas corresponderam a RS 295,8 milhões $(77,1 \%)$; em ciências sociais, $\mathrm{R}$ \$ 43,1 milhões (11,2\%), e R\$ 17,7 milhões em ciências naturais $(4,6 \%)$. Investimentos em infraestrutura de C\&T/S perfilaram $3,9 \%$, enquanto $\mathrm{R} \$ 12,3$ milhões $(3,2 \%)$ corresponderam a pesquisas relativas a outros campos, como aquelas relacionadas a investigações sobre novas tecnologias de saneamento, modelos e metodologias para investigar impacto ambiental de projetos etc.

O levantamento dos recursos investidos em $\mathrm{P} \& \mathrm{D} / \mathrm{S}$ permitiu a constituição de uma matriz fonte-usuário, que relaciona a origem e o destino dos recursos empregados em $\mathrm{P} \& \mathrm{D} / \mathrm{S}$, no caso a partir dos dispêndios do Ministério da Saúde, mostrada na Figura 1.

A classificação pela ANPPS levou em conta o primeiro nível das subagendas, com as pesquisas sendo classificadas em até três itens. Em decorrência, o volume correspondente ao somatório dos dispêndios aplicados a cada item supera os R\$ 409,7 milhões investidos no período pelo Ministério da Saúde. Interessa, contudo, conhecer o volume total aplicado a cada subagenda, sendo o percentual correspondente calculado em função do valor total efetivamente aplicado pelo Ministério no triênio (Tabela 1).

Dois pontos importantes merecem destaque. $\mathrm{O}$ primeiro se refere a $\mathrm{R} \$ 52,2$ milhões efetivamente gastos com $\mathrm{P} \& \mathrm{D} / \mathrm{S}$ no triênio, onde não foi possível identificar, de forma precisa, o objeto da pesquisa, impossibilitando sua categorização

Figura 1

Fluxos financeiros em pesquisa e desenvolvimento em saúde (P\&D/S) do Ministério da Saúde segundo categoria institucional da usuária dos recursos no período de 2003-2005.
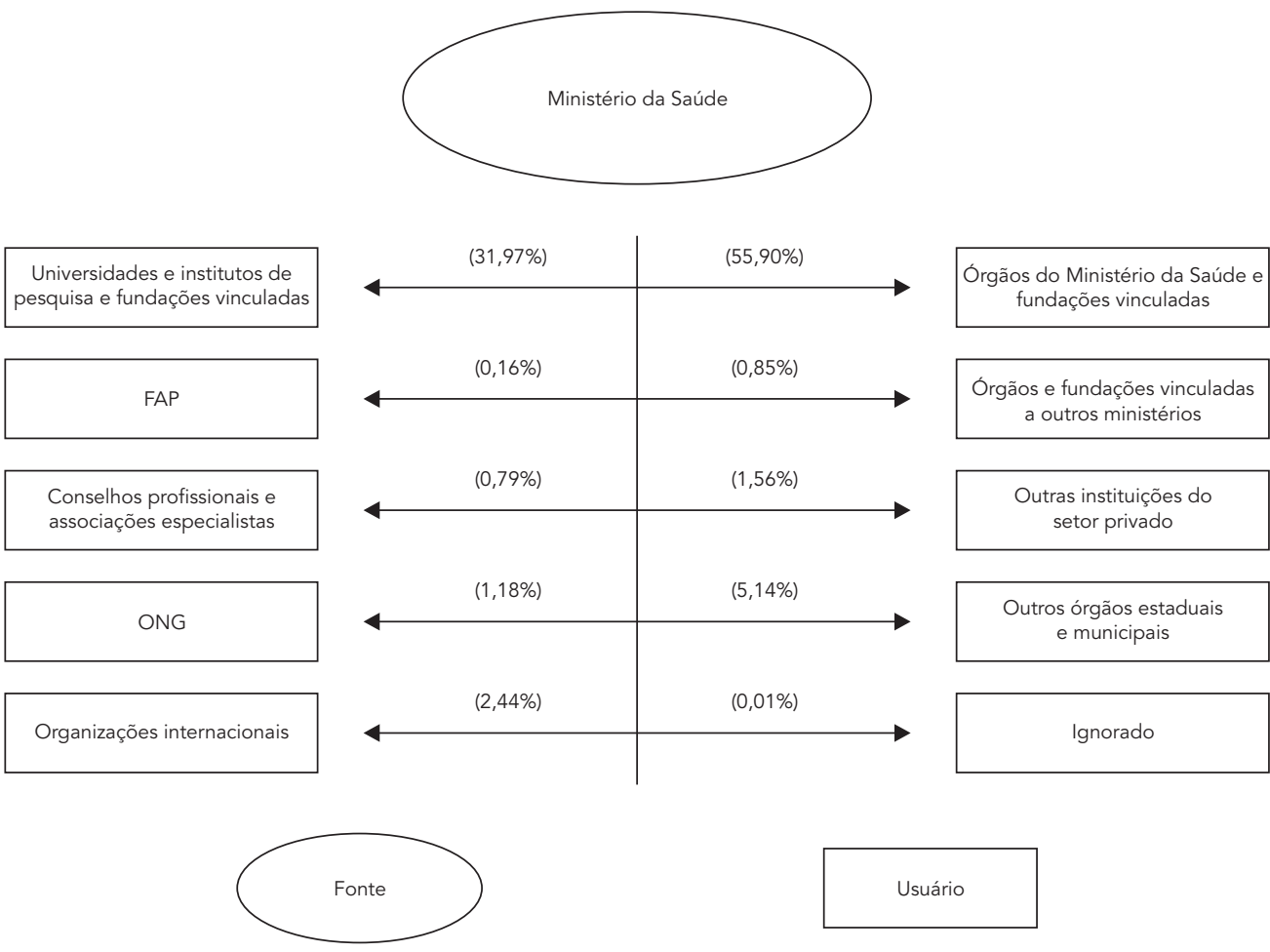

Usuário

FAP: Fundações de Amparo à Pesquisa; ONG: organizações não-governamentais.

Nota: as formas geométricas no limite inferior da figura visam informar a classificação de cada categoria institucional,

segundo sua posição nos fluxos de recursos financeiros especificados. 
Dispêndios em pesquisa e desenvolvimento em saúde (P\&D/S) do Ministério da Saúde segundo as subagendas da Agenda Nacional de Prioridades de Pesquisa em Saúde (ANPPS), no período de 2003-2005 (em R\$).

\begin{tabular}{|c|c|c|c|c|c|}
\hline Subagenda & 2003 & 2004 & 2005 & 2003-2005 & $\%$ * \\
\hline 19. Doenças transmissíveis & $19.414 .665,74$ & $44.817 .481,79$ & $73.867 .100,73$ & $138.099 .248,26$ & 33,7 \\
\hline 13. Complexo produtivo da saúde & $8.080 .225,77$ & $19.277 .680,28$ & $31.384 .758,29$ & $58.742 .664,34$ & 14,3 \\
\hline 12. Pesquisa clínica & $623.656,20$ & 7.317.145,28 & $41.959 .674,79$ & $49.900 .476,28$ & 12,2 \\
\hline 24. Assistência farmacêutica & $4.027 .738,34$ & $8.804 .724,34$ & $33.389 .047,89$ & $46.221 .510,57$ & 11,3 \\
\hline 5. Doenças não-transmissíveis & $6.600 .266,45$ & $3.503 .205,98$ & $20.918 .447,06$ & $31.021 .919,49$ & 7,6 \\
\hline 23. Saúde, ambiente, trabalho e biossegurança & $10.024 .358,60$ & $11.514 .856,66$ & $5.272 .044,46$ & $26.811 .259,72$ & 6,5 \\
\hline 22. Sistemas e políticas de saúde & $5.789 .193,98$ & $9.515 .158,73$ & $7.891 .053,52$ & $23.195 .406,24$ & 5,7 \\
\hline 14. Avaliação de tecnologias e economia da saúde & $7.274 .661,64$ & $4.539 .759,21$ & $8.298 .855,79$ & $20.113 .276,64$ & 4,9 \\
\hline 10. Alimentação e nutrição & $1.755 .749,43$ & $7.393 .165,22$ & $6.777 .201,16$ & $15.926 .115,82$ & 3,9 \\
\hline 7. Saúde da criança e do adolescente & $1.140 .465,28$ & $3.697 .780,45$ & $6.700 .265,53$ & $11.538 .511,27$ & 2,8 \\
\hline 8. Saúde da mulher & $801.830,20$ & $1.860 .980,37$ & $6.364 .741,55$ & $9.027 .552,12$ & 2,2 \\
\hline 16. Demografia e saúde & 0,00 & 0,00 & $8.045 .744,57$ & $8.045 .744,57$ & 2,0 \\
\hline 15. Epidemiologia & $921.038,35$ & $835.591,67$ & $5.980 .516,22$ & $7.737 .146,24$ & 1,9 \\
\hline 11. Bioética e etica na pesquisa & $354.981,87$ & $1.237 .458,27$ & $5.396 .843,25$ & $6.989 .283,39$ & 1,7 \\
\hline 3. Violência, acidentes e trauma & $305.039,49$ & $2.779 .125,27$ & $3.469 .860,31$ & $6.554 .025,08$ & 1,6 \\
\hline 2. Saúde mental & $329.721,60$ & $512.527,32$ & $4.661 .954,14$ & $5.504 .203,07$ & 1,3 \\
\hline 1. Saúde dos povos indígenas & $93.899,80$ & $999.640,60$ & $2.303 .371,72$ & $3.396 .912,11$ & 0,8 \\
\hline 6. Saúde do idoso & $197.025,94$ & $366.617,22$ & $1.451 .997,95$ & $2.015 .641,12$ & 0,5 \\
\hline 20. Comunicação e informação em saúde & $625.890,02$ & $854.306,22$ & $525.903,93$ & $2.006 .100,17$ & 0,5 \\
\hline 18. Promoção da saúde & $854.394,14$ & $396.838,11$ & $545.013,59$ & $1.796 .245,83$ & 0,4 \\
\hline 21. Gestão do trabalho e educação em saúde & $405.395,60$ & $587.890,13$ & $648.899,01$ & $1.642 .184,74$ & 0,4 \\
\hline 17. Saúde bucal & $149.348,60$ & $498.727,54$ & $663.257,33$ & $1.311 .333,47$ & 0,3 \\
\hline 4. Saúde da população negra & 0,00 & 0,00 & $1.102 .093,38$ & $1.102 .093,38$ & 0,3 \\
\hline 9. Saúde dos portadores de necessidades especiais & $21.760,00$ & $223.133,55$ & $603.814,72$ & $848.708,27$ & 0,2 \\
\hline Pesquisas com objeto identificado fora da ANPPS & $1.813 .918,32$ & $5.063 .220,20$ & $5.376 .234,79$ & $12.253 .373,31$ & 3,0 \\
\hline
\end{tabular}

* As porcentagens foram calculadas tendo como denominador o valor total de recursos investidos, de modo que a soma das porcentagens ultrapassa $100 \%$.

segundo as subagendas da ANPPS. É o caso, por exemplo, de financiamentos procedentes do antigo Centro Nacional de Epidemiologia e do Departamento de Engenharia Sanitária, ambos vinculados à FUNASA, e cujas informações apenas permitem a identificação aproximada de seu objeto, com alguns deles sendo caracterizados como "estudos e pesquisas", mas sem que se discriminem os projetos de pesquisas englobados nos convênios celebrados. Outro aspecto corresponde a dispêndios onde era identificado o objeto de pesquisa, mas este não possibilitava sua classificação nas subagendas. Estas pesquisas foram categorizadas como "pesquisas com objeto identificado fora da agenda de prioridades de pesquisa" e corresponderam a cerca de $3 \%$ do total de gastos do Ministério da Saúde em P\&D/S.

O maior aporte de recursos foi aplicado na subagenda doenças transmissíveis $(33,7 \%)$, seguidos do complexo produtivo da saúde (14,3\%); pesquisa clínica (12,2\%); assistência farmacêutica
$(11,3 \%)$ e doenças não-transmissíveis $(7,6 \%)$. As subagendas saúde dos portadores de necessidades especiais $(0,2 \%)$ e saúde da população negra $(0,3 \%)$ apresentaram os menores investimentos.

Segundo a região de execução das pesquisas, é verificada a relevância do Sudeste em quase todas as subagendas. No caso das subagendas saúde da população negra e saúde da população indígena, a região de maior presença de esforço de pesquisa foi a Norte. Já para a subagenda saúde bucal, foi verificada uma distribuição mais homogênea entre as várias regiões.

As subagendas mais predominantes em cada região do país (Figura 2) tendem a refletir as características das estruturas de pesquisa nelas presente. Por exemplo, na Região Norte, a alta concentração de pesquisas na área das doenças transmissíveis $(53,6 \%)$ possivelmente se relaciona à presença de três Centros de Pesquisa, da Fiocruz e da Secretaria de Vigilância à Saúde, que desenvolvem investigações em temas relaciona- 
Dispêndios em pesquisa e desenvolvimento em saúde (P\&D/S) do Ministério da Saúde segundo as subagendas da Agenda Nacional de Prioridades de Pesquisa em Saúde (ANPPS), predominantes por região brasileira, no período de 2003-2005.

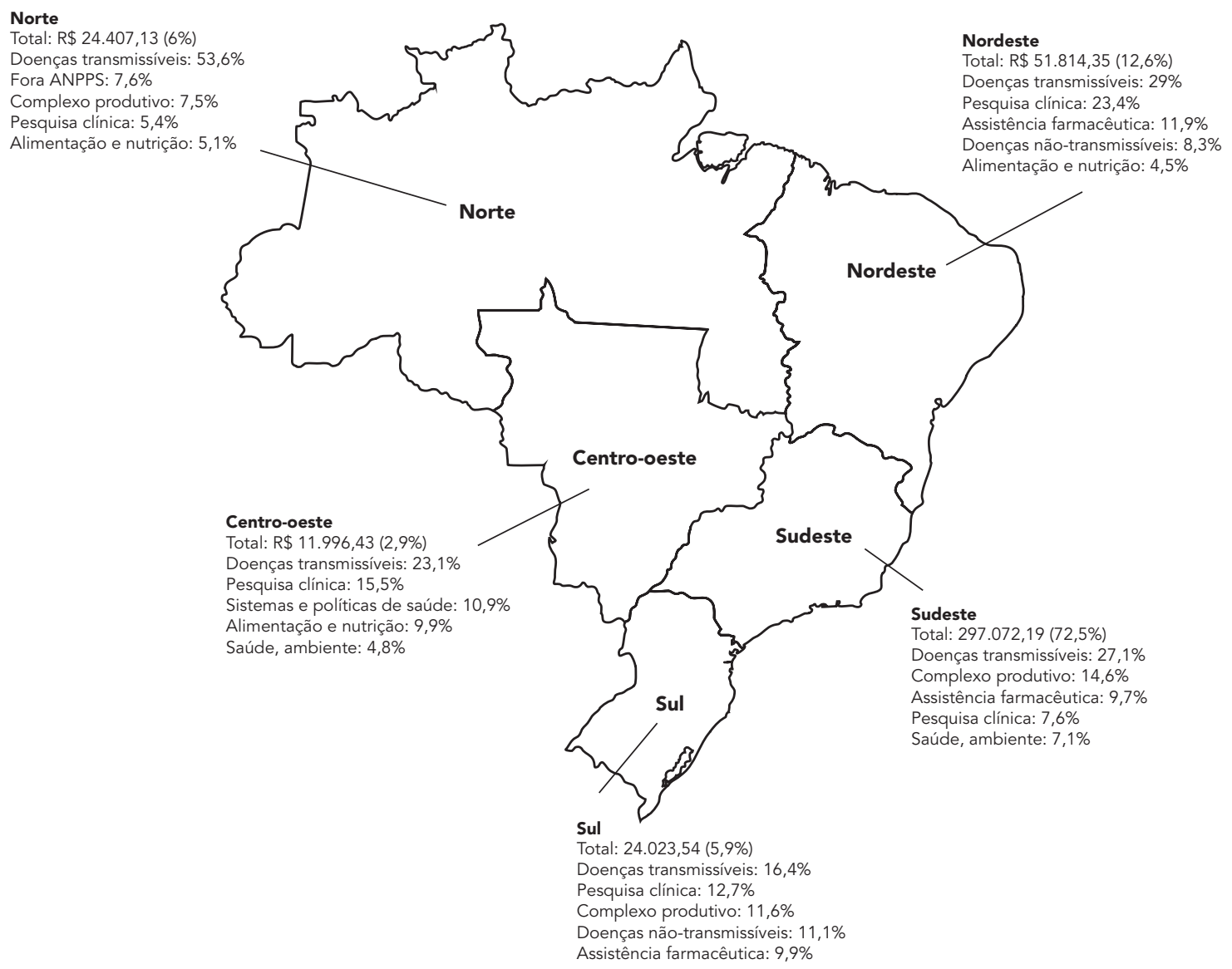

Nota: os totais referem-se ao volume de investimentos em P\&D/S de cada região em milhões de reais, enquanto os percentuais relacionam-se ao percentual de recursos relativos às cinco subagendas predominantes nas mesmas.

dos a doenças tropicais endêmicas na Amazônia, dentre outros.

Em todas as regiões, observa-se marcante presença da subagenda doenças transmissíveis. O Sul apresenta a distribuição mais homogênea dentre todas as regiões, ainda que manifeste o mesmo predomínio dos investimentos relativos à subagenda citada. Nas demais, alternam-se as posições relativas aos postos subsequentes. No Centro-oeste, as outras duas principais Subagendas em termos de financiamento são as de pesquisas clinicas e de sistemas e políticas de saúde, destacando-se em relação a esta última o volume de financiamentos destinados a Universidade de Brasília. No Nordeste, a assistência farmacêutica figura como importante rubrica, em parte de- corrente da intensidade de pesquisas presentes no Ceará, com tradição de pesquisa na área de medicamentos. No Sudeste, destaca-se o volume de financiamentos relacionados ao complexo produtivo da saúde, alavancado pelas pesquisas desenvolvidas na Fiocruz.

$\mathrm{Na}$ distribuição dos investimentos nas Subagendas estratificada pela fonte (Tabela 2), segundo o tipo de administração do Ministério da Saúde, observa-se o foco da Administração Indireta no fomento à subagenda das doenças transmissíveis $(60,5 \%)$, explicado em grande parte pelos estudos da Fiocruz nesta área. A subagenda pesquisa clínica apresenta investimentos oriundos da Administração Direta (83\%), que provêm predominantemente do DECIT/SCTIE, 
Investimentos em pesquisa e desenvolvimento em saúde (P\&D/S) do Ministério da Saúde segundo as subagendas da Agenda Nacional de Prioridades de Pesquisa em Saúde (ANPPS), e órgão-fonte do financiamento no período de 2003-2005 (em \%).

\begin{tabular}{|c|c|c|c|c|c|c|c|c|c|}
\hline \multirow[t]{2}{*}{ Subagenda } & \multicolumn{4}{|c|}{ Administração indireta } & \multicolumn{4}{|c|}{ Administração direta } & \multirow[t]{2}{*}{ Total (\%) } \\
\hline & ANS & ANVISA & FIOCRUZ & FUNASA & SE & SAS & SVS & SCTIE & \\
\hline 19. Doenças transmissíveis & 0,0 & 0,0 & 60,2 & 0,3 & 6,0 & 0,0 & 8,3 & 25,2 & 33,7 \\
\hline 13. Complexo produtivo da saúde & 0,0 & 0,5 & 51,5 & 0,0 & 8,3 & 0,0 & 2,3 & 37,4 & 14,3 \\
\hline 12. Pesquisa clínica & 0,0 & 0,0 & 17,0 & 0,0 & 3,6 & 0,0 & 0,8 & 78,6 & 12,0 \\
\hline 24. Assistência farmacêutica & 0,0 & 5,1 & 55,6 & 0,0 & 3,4 & 0,0 & 5,3 & 30,6 & 11,1 \\
\hline 5. Doenças não-transmissíveis & 0,0 & 1,3 & 22,7 & 0,0 & 14,8 & 2,5 & 2,1 & 56,6 & 7,6 \\
\hline 23. Saúde, ambiente, trabalho e biossegurança & 0,0 & 36,5 & 51,3 & 2,8 & 1,2 & 0,0 & 0,0 & 8,2 & 6,5 \\
\hline 22. Sistemas e políticas de saúde & 23,1 & 0,0 & 35,7 & 0,0 & 6,0 & 0,0 & 0,1 & 35,0 & 5,7 \\
\hline 14. Avaliação de tecnologias e economia da saúde & 11,6 & 11,5 & 32,6 & 0,0 & 8,3 & 0,0 & 1,1 & 34,9 & 4,9 \\
\hline 10. Alimentação e nutrição & 0,0 & 23,2 & 21,5 & 0,4 & 26,6 & 0,0 & 0,0 & 28,3 & 3,9 \\
\hline 7. Saúde da criança e do adolescente & 0,0 & 0,0 & 46,3 & 1,7 & 5,8 & 1 & 6,3 & 38,9 & 2,8 \\
\hline 8. Saúde da mulher & 0,0 & 0,0 & 41,7 & 0,0 & 2,5 & 0,0 & 2,8 & 53,0 & 2,2 \\
\hline 16. Demografia e saúde & 0,0 & 0,0 & 0,0 & 0,0 & 0,0 & 0,0 & 3,4 & 96,6 & 2,0 \\
\hline 15. Epidemiologia & 0,0 & 0,0 & 32,4 & 0,0 & 29,1 & 0,0 & 0,4 & 38,1 & 1,9 \\
\hline 11. Bioética e ética na pesquisa & 0,0 & 0,0 & 56,3 & 0,0 & 0,0 & 0,0 & 3,9 & 39,8 & 1,7 \\
\hline 3. Violência, acidentes e trauma & 0,0 & 0,0 & 23,8 & 0,0 & 4,5 & 0,0 & 1,7 & 70,1 & 1,6 \\
\hline 2. Saúde mental & 0,0 & 0,0 & 8,4 & 0,4 & 9,5 & 0,0 & 0,0 & 81,7 & 1,3 \\
\hline 1. Saúde dos povos indígenas & 0,0 & 0,0 & 38,1 & 3,5 & 0,0 & 0,0 & 0,0 & 58,5 & 0,8 \\
\hline 6. Saúde do idoso & 0,0 & 0,0 & 40,4 & 0,0 & 0,0 & 0,0 & 0,0 & 59,6 & 0,5 \\
\hline 20. Comunicação e informação em saúde & 46,0 & 0,0 & 22,5 & 0,0 & 0,0 & 0,0 & 1,3 & 30,3 & 0,5 \\
\hline 18. Promoção da saúde & 0,0 & 0,0 & 60,9 & 0,6 & 0,0 & 0,0 & 8,8 & 29,7 & 0,4 \\
\hline 21. Gestão do trabalho e educação em saúde & 0,0 & 0,0 & 13,5 & 0,0 & 14,9 & 0,0 & 1,6 & 70,0 & 0,4 \\
\hline 17. Saúde bucal & 0,0 & 0,0 & 0,5 & 0,8 & 0,0 & 0,0 & 0,0 & 98,7 & 0,3 \\
\hline 4. Saúde da população negra & 0,0 & 0,0 & 49,1 & 0,0 & 0,0 & 0,0 & 26,1 & 24,8 & 0,3 \\
\hline 9. Saúde dos Portadores de necessidades especiais & 0,0 & 0,0 & 4,6 & 0,0 & 0,0 & 0,0 & 0,0 & 95,4 & 0,2 \\
\hline Pesquisas com objeto identificado fora da ANPPS & 0,0 & 22,2 & 45,4 & 0,0 & 4,7 & 0,0 & 23,0 & 4,7 & 3,0 \\
\hline
\end{tabular}

ANS: Agência Nacional de Saúde Suplementar; ANVISA: Agência Nacional de Vigilância Sanitária; Fiocruz: Fundação Oswaldo Cruz; FUNASA: Fundação Nacional de Saúde; SAS: Secretaria de Assistência à Saúde; SCTIE: Secretaria de Ciência, Tecnologia e Insumos Estratégicos; SE: Secretaria Executiva; SVS: Secretaria de Vigilância em Saúde.

parte dos quais relacionados aos editais temáticos de células-tronco. Outro ponto refere-se ao volume de investimentos da Administração Indireta na subagenda assistência farmacêutica (60,7\%), motivados pela presença importante da Fiocruz, via Farmanguinhos e Bio-Manguinhos, além de outras linhas de pesquisa no tema. Nas doenças não-transmissíveis, é marcante a presença da Administração Direta (76\%), decorrente da presença de financiamentos oriundos da Secretaria de Assistência a Saúde e de alguns editais temáticos com recursos da SCTIE, como por exemplo, o de neoplasias e do Estudo Multicêntrico Longitudinal em Doenças Cardiovasculares e Diabetes Mellitus/"ELSA". Nesta mesma linha, com investimentos alavancados por editais temáticos do DECIT/SCTIE, tem-se o predomínio da Administração Direta nas Subagendas saúde dos povos indígenas; saúde mental; violência, acidentes e trauma; e demografia (consequente ao estudo Pesquisa Nacional de Demografia, Saúde da Criança e da Mulher).

A Tabela 3 apresenta a distribuição proporcional dos dispêndios por subagenda segundo a categoria institucional da executora dos recursos. É elevado o percentual de financiamentos na subagenda doenças transmissíveis recebidos prioritariamente por órgãos e fundações do Ministério da Saúde (74\%). No caso do complexo produtivo da saúde, a distribuição segue caminho semelhante (71,3\%). Em ambos os casos, destaca-se a presença do complexo Fiocruz, com diversas linhas de pesquisa nestes temas. A subagenda pesquisa clínica apresenta as universidades e institutos de pesquisas $(58,5 \%)$ como principais receptores dos investimentos, visto que foram os que mais 
Dispêndios em pesquisa e desenvolvimento em saúde (P\&D/S) do Ministério da Saúde segundo as subagendas da Agenda Nacional de Prioridades de Pesquisa em Saúde (ANPPS), e categoria institucional da executora das pesquisas no período de $2003-2005$ (em \%).

\begin{tabular}{|c|c|c|c|c|c|c|c|c|c|c|c|}
\hline Subagenda & $\begin{array}{l}\text { Órgãos do } \\
\text { Ministério } \\
\text { da Saúde e } \\
\text { fundações } \\
\text { vinculadas }\end{array}$ & $\begin{array}{l}\text { Órgãos e } \\
\text { fundações } \\
\text { vinculadas } \\
\text { a outros } \\
\text { ministérios }\end{array}$ & FAP & $\begin{array}{c}\text { Outros } \\
\text { órgãos } \\
\text { estaduais e } \\
\text { municipais }\end{array}$ & ONG & $\begin{array}{l}\text { Conselhos } \\
\text { profissionais } \\
\text { e asso- } \\
\text { ciações de } \\
\text { especialistas }\end{array}$ & $\begin{array}{l}\text { Universidades } \\
\text { e institutos } \\
\text { de pesquisa } \\
\text { e fundações } \\
\text { vinculadas }\end{array}$ & $\begin{array}{l}\text { Outras } \\
\text { instituições } \\
\text { do setor } \\
\text { privado }\end{array}$ & $\begin{array}{l}\text { Organiza- } \\
\text { ções } \\
\text { interna- } \\
\text { cionais }\end{array}$ & Ignorado & $\%$ \\
\hline $\begin{array}{l}\text { 19. Doenças } \\
\text { transmissíveis }\end{array}$ & 74,0 & 0,5 & 0,0 & 0,1 & 1,5 & 0,2 & 17,5 & 0,3 & 0,0 & 0,0 & 33,7 \\
\hline $\begin{array}{l}\text { 13. Complexo } \\
\text { produtivo da saúde }\end{array}$ & 71,3 & 0,2 & 0,0 & 0,1 & 4,6 & 0,0 & 10,4 & 1,8 & 0,0 & 0,1 & 14,3 \\
\hline 12. Pesquisa clínica & 30,1 & 0,0 & 0,0 & 0,0 & 0,1 & 1,5 & 58,5 & 7,1 & 0,0 & 0,0 & 12,2 \\
\hline $\begin{array}{l}\text { 24. Assistência } \\
\text { farmacêutica }\end{array}$ & 66,1 & 1,1 & 0,1 & 0,0 & 0,0 & 0,8 & 31,0 & 0,3 & 0,0 & 0,1 & 11,3 \\
\hline $\begin{array}{l}\text { 5. Doenças não- } \\
\text { transmissíveis }\end{array}$ & 39,4 & 0,0 & 0,5 & 0,0 & 0,1 & 0,6 & 55,5 & 0,8 & 0,0 & 0,0 & 7,6 \\
\hline $\begin{array}{l}\text { 23. Saúde, } \\
\text { ambiente, trabalho e } \\
\text { biossegurança }\end{array}$ & 68,0 & 0,0 & 0,3 & 0,1 & 0,4 & 1,5 & 15,2 & 0,2 & 0,0 & 0,0 & 6,5 \\
\hline $\begin{array}{l}\text { 22. Sistemas e } \\
\text { políticas de saúde }\end{array}$ & 46,6 & 0,0 & 0,8 & 0,0 & 2,2 & 0,0 & 48,3 & 0,4 & 0,0 & 0,0 & 5,7 \\
\hline $\begin{array}{l}\text { 14. Avaliação de } \\
\text { tecnologias e } \\
\text { economia da saúde }\end{array}$ & 43,3 & 2,1 & 0,0 & 0,0 & 0,5 & 0,3 & 48,9 & 1,1 & 0,0 & 0,0 & 4,9 \\
\hline $\begin{array}{l}\text { 10. Alimentação e } \\
\text { nutrição }\end{array}$ & 28,4 & 0,4 & 0,0 & 0,3 & 0,0 & 1,3 & 40,0 & 0,1 & 0,0 & 0,0 & 3,9 \\
\hline $\begin{array}{l}\text { 7. Saúde da criança } \\
\text { e do adolescente }\end{array}$ & 60,2 & 0,5 & 0,4 & 0,0 & 1,0 & 1,8 & 32,5 & 1,7 & 0,0 & 0,0 & 2,8 \\
\hline 8. Saúde da mulher & 52,5 & 0,6 & 0,2 & 0,0 & 1,6 & 2,6 & 41,0 & 0,8 & 0,0 & 0,0 & 2,2 \\
\hline $\begin{array}{l}\text { 16. Demografia e } \\
\text { saúde }\end{array}$ & 0,0 & 0,0 & 0,0 & 0,0 & 0,0 & 0,0 & 96,6 & 3,4 & 0,0 & 0,0 & 2,0 \\
\hline 15. Epidemiologia & 39,4 & 0,0 & 0,0 & 0,0 & 0,2 & 2,5 & 56,9 & 0,0 & 0,0 & 0,0 & 1,9 \\
\hline $\begin{array}{l}\text { 11. Bioética e ética } \\
\text { na pesquisa }\end{array}$ & 59,4 & 0,0 & 0,2 & 0,0 & 7,3 & 0,4 & 26,4 & 4,4 & 0,0 & 0,0 & 1,7 \\
\hline $\begin{array}{l}\text { 3. Violência, } \\
\text { acidentes e trauma }\end{array}$ & 35,6 & 0,0 & 0,9 & 0,0 & 0,0 & 3,2 & 55,6 & 0,0 & 0,0 & 0,0 & 1,6 \\
\hline 2. Saúde mental & 9,4 & 0,0 & 0,0 & 0,0 & 0,0 & 3,1 & 87,0 & 0,0 & 0,0 & 0,0 & 1,3 \\
\hline $\begin{array}{l}\text { 1. Saúde dos povos } \\
\text { indígenas }\end{array}$ & 51,8 & 0,0 & 1,2 & 0,0 & 0,9 & 0,0 & 46,1 & 0,0 & 0,0 & 0,0 & 0,8 \\
\hline 6. Saúde do idoso & 52,9 & 0,0 & 0,6 & 0,0 & 0,0 & 0,0 & 45,5 & 0,0 & 0,0 & 0,0 & 0,5 \\
\hline $\begin{array}{l}\text { 20. Comunicação } \\
\text { e informação em } \\
\text { saúde }\end{array}$ & 23,5 & 1,0 & 1,1 & 0,0 & 1,3 & 0,0 & 41,0 & 27,5 & 0,0 & 0,0 & 0,5 \\
\hline $\begin{array}{l}\text { 18. Promoção da } \\
\text { saúde }\end{array}$ & 64,1 & 0,0 & 0,9 & 0,0 & 2,5 & 0,0 & 30,4 & 1,9 & 0,0 & 0,0 & 0,4 \\
\hline $\begin{array}{l}\text { 21. Gestão do } \\
\text { trabalho e educação } \\
\text { em saúde }\end{array}$ & 13,5 & 0,0 & 0,0 & 0,0 & 1,6 & 3,0 & 78,9 & 3,0 & 0,0 & 0,0 & 0,4 \\
\hline 17. Saúde bucal & 0,5 & 0,0 & 0,0 & 0,1 & 0,0 & 0,0 & 86,8 & 1,6 & 0,0 & 0,0 & 0,3 \\
\hline $\begin{array}{l}\text { 4. Saúde da } \\
\text { população negra }\end{array}$ & 73,9 & 0,0 & 0,0 & 0,0 & 0,0 & 0,0 & 26,1 & 0,0 & 0,0 & 0,0 & 0,3 \\
\hline
\end{tabular}

(continua) 
Tabela 3 (continuação)

\begin{tabular}{|c|c|c|c|c|c|c|c|c|c|c|c|}
\hline Subagenda & $\begin{array}{l}\text { Órgãos do } \\
\text { Ministério } \\
\text { da Saúde e } \\
\text { fundações } \\
\text { vinculadas }\end{array}$ & $\begin{array}{l}\text { Órgãos e } \\
\text { fundações } \\
\text { vinculadas } \\
\text { a outros } \\
\text { ministérios }\end{array}$ & FAP & $\begin{array}{c}\text { Outros } \\
\text { órgãos } \\
\text { estaduais e } \\
\text { municipais }\end{array}$ & ONG & $\begin{array}{l}\text { Conselhos } \\
\text { profissionais } \\
\text { e asso- } \\
\text { ciações de } \\
\text { especialistas }\end{array}$ & $\begin{array}{l}\text { Universidades } \\
\text { e institutos } \\
\text { de pesquisa } \\
\text { e fundações } \\
\text { vinculadas }\end{array}$ & $\begin{array}{l}\text { Outras } \\
\text { instituições } \\
\text { do setor } \\
\text { privado }\end{array}$ & $\begin{array}{l}\text { Organiza- } \\
\text { ções } \\
\text { interna- } \\
\text { cionais }\end{array}$ & Ignorado & $\%$ \\
\hline $\begin{array}{l}\text { 9. Saúde dos } \\
\text { portadores de } \\
\text { necessidades } \\
\text { especiais }\end{array}$ & 16,3 & 0,0 & 0,0 & 0,0 & 28,8 & 30,6 & 24,3 & 0,0 & 0,0 & 0,0 & 0,2 \\
\hline $\begin{array}{l}\text { Pesquisas com } \\
\text { objeto identificado } \\
\text { fora da ANPPS }\end{array}$ & 48,5 & 0,0 & 0,0 & 0,0 & 0,0 & 2,3 & 47,9 & 0,0 & 0,0 & 0,0 & 3,0 \\
\hline
\end{tabular}

FAP: Fundações de Amparo à Pesquisa; ONG: organizações não-governamentais.

auferiram recursos dos editais direcionados à implantação de unidades de pesquisa clínica em hospitais universitários e a investigações na área de células-tronco. O mesmo padrão de concentração é observado na subagenda doenças nãotransmissíveis $(55,5 \%)$, resultante, entre outros, dos significativos volumes associados ao Estudo ELSA e ao Estudo Multicêntrico Randomizado de Terapia Celular em Cardiopatias, voltado ao desenvolvimento de pesquisa de implantes autólogos de células-tronco. A subagenda saúde dos portadores de necessidades especiais apresenta distribuição diferente, com conselhos profissionais e associações de especialistas recebendo $30,6 \%$ dos recursos, secundados pelas organizações não-governamentais $(28,8 \%)$.

A distribuição dos investimentos pelo segundo nível das subagendas mostra que dez subníveis representam pouco mais de $70 \%$ do volume total investido no período: (1) novos conhecimentos em doenças infecciosas; (2) novos instrumentos em doenças infecciosas; (3) inovação e desenvolvimento tecnológico em vacinas; (4) temas específicos em pesquisa clínica; (5) desenvolvimento e avaliação de farmoquímicos e medicamentos; (6) neoplasias; (7) prioridades comuns ao conjunto das doenças não-transmissíveis relevantes para a saúde pública; (8) avaliação das políticas públicas regulatórias do estado em relação à saúde, ambiente, trabalho e biossegurança; (9) dinâmica e compreensão dos sistemas e políticas de saúde; e, (10) ciclo de vida de tecnologias em saúde (inovação, incorporação, uso e obsolescência).

Novos conhecimentos em doenças transmissíveis correspondem ao item de segundo nível hierárquico da ANPPS que recebeu maior investimento no período, incluindo pesquisas voltadas a condições com tendência declinante em nosso meio (doenças imunopreveníveis, doença de Chagas, hanseníase) até outras que persistem de forma expressiva (tuberculose, malária, leishmanioses) e doenças emergentes e reemergentes (cólera, dengue, hantavirose).

Outro subitem expressivo foi o de inovação e desenvolvimento tecnológico em vacinas, da subagenda complexo produtivo da saúde. Investimentos significativos estiveram voltados à P\&D de vacinas prioritárias no contexto sanitário nacional, incluindo os projetos INOVABIO, objetivando o desenvolvimento de seis vacinas prioritárias para o Programa Nacional de Imunizações, e INOVACINA, elaborado para incentivar a produção de vacinas pelos laboratórios públicos do país e, consequentemente, minimizar a dependência de importação de insumos importantes para a saúde pública.

Temas específicos em pesquisa clínica, constituinte da subagenda pesquisa clínica, também recebeu investimentos representativos, ressaltando-se o apoio à organização da Rede Nacional de Pesquisa Clínica, objetivando disponibilizar infraestrutura básica para o desenvolvimento de todas as fases de ensaios clínicos de fármacos, procedimentos, equipamentos e dispositivos para diagnósticos. Outro aspecto relevante refere-se às pesquisas em células-tronco, que se constituem como setor de ponta na pesquisa em saúde mundial.

\section{Discussão}

Observando a evolução dos investimentos do Ministério da Saúde no período, destaca-se o fortalecimento de seu papel de formulador e gestor da política de C\&T. Registre-se que o volume de recursos próprios para este financiamento pelo 
Ministério da Saúde só começou a ascender fortemente após a criação do DECIT em 2000 e, em particular, após a estruturação da SCTIE em 2003 6,14. Com o estabelecimento do departamento, a C\&T/S ganha novo status na estrutura do Ministério, passando a ter um orçamento específico e contínuo para essa área, ampliando as perspectivas para o setor.

No cumprimento do papel como financiador da $\mathrm{P} \& \mathrm{D} / \mathrm{S}$ no país, o Ministério da Saúde, só em termos do DECIT/SCTIE, apresentou um incremento no volume de recursos entre 2003 e 2005, respectivamente, $\mathrm{R} \$ 6,7$ milhões; $\mathrm{R} \$ 60,2$ milhões e R\$ 127,8 milhões 11. Esses volumes, contudo, correspondem a valores orçamentários comprometidos - mas não necessariamente aplicados nesses exatos períodos de tempo, diferentemente do que foi aferido neste trabalho.

Também neste foi possível observar elevação nos recursos investidos anualmente pelo Ministério da Saúde no financiamento da $\mathrm{P} \& \mathrm{D} / \mathrm{S}$ : de R\$ 88,2 milhões em 2003, para R\$ 207,6 milhões, em 2005. Se compararmos aos volumes de financiamento ministerial de 2000-2002 15, verifica-se que o total de recursos investidos elevou-se de R\$ 199 milhões para cerca de R $\$ 110$ milhões, entre 2003-2005, representando aumento de mais de $100 \%$ nos investimentos (excluídos os salários de suas instituições próprias de pesquisa). Esse dado já aponta o papel significativo assumido pelo Ministério da Saúde no cenário de fomento à pesquisa neste século, no qual possuem relevância os volumes investidos pela SCTIE e os orçamentos próprios de pesquisa da Fiocruz.

Não é suficiente, contudo, apenas o aumento nos recursos destinados a $\mathrm{P} \& \mathrm{D} / \mathrm{S}$ para direcionar as pesquisas e orientar a implementação da PNCTI/S. O fomento precisa seguir linhas definidas e prioritárias, com temas de interesse para a construção de políticas de C\&T/S voltadas para a solução de problemas e necessidades de saúde da população, e melhoria da realidade do SUS. Na medida em que parte desse investimento do Ministério da Saúde já possa estar "orientada” pela ANPPS, caminhar-se-ia, ainda que de forma bem preliminar, para reduzir o hiato já apontado.

Apesar da Agenda ainda ser bastante recente, a análise dos resultados de investimentos frente às suas subagendas constituintes permitiu identificar um baixo índice de dispêndios em temas fora da ANPPS (apenas 3\% do total de recursos aplicados). Não é possível afirmar as explicações para este percentual. Por um lado, pode deverse ao caráter muito abrangente e inclusivo da ANPPS, principalmente quando observado sob a ótica de seu primeiro nível hierárquico. Pouquíssimas questões podem ser identificadas como explicitamente fora da Agenda, tais como a saúde da população masculina e genética clínica. Por outro, pode estar indicando que os financiamentos em P\&D/S do Ministério da Saúde ocorridos no triênio já se relacionavam, de alguma forma, às prioridades estabelecidas em 2004, uma vez que, no período 2003-2005, o DECIT/SCTIE lançou diversos editais e chamadas públicas para apresentação de propostas para posterior financiamento de pesquisas que já contemplavam subagendas específicas da ANPPS 6 .

Em termos da distribuição dos recursos segundo as subagendas, os dados apontaram para uma grande participação de cinco subagendas principais - doenças transmissíveis, complexo produtivo da saúde, pesquisa clínica, assistência farmacêutica e doenças não-transmissíveis - que, somadas, representaram investimentos da ordem de R \$ 324 milhões entre 2003-2005.

Assinale-se o fato de que todas as subagendas apresentarem algum grau de investimento no triênio, ainda que, em alguns casos, de volume pouco significativo, como é o caso da saúde dos portadores de necessidades especiais, saúde da população negra, e saúde bucal. Essa situação, entretanto, representa uma evolução em termos do período 2000-2002, quando, por exemplo, a subagenda saúde da população negra não recebeu qualquer investimento ${ }^{15}$.

Merece menção o fato de que a opção, iniciada no período, de financiamentos via editais temáticos insere a possibilidade de distorções na distribuição dos recursos segundo as várias subagendas. Esta modalidade passa a ser preponderante na atuação do Ministério da Saúde, especialmente no DECIT, a partir de 2004, direcionando os investimentos para áreas específicas. Por outro lado, instituições internas ao Ministério da Saúde com orçamento próprio de pesquisa, como é o caso da Fiocruz, a maior financiadora no triênio, com autonomia na destinação de seus recursos de $\mathrm{P} \& \mathrm{D}$, podem vir a utilizá-los sem estar necessariamente direcionadas pelas prioridades presentes na ANPPS.

Faz-se necessário assinalar algumas dificuldades e limitações do estudo empreendido. $\mathrm{O}$ universo de financiamentos estudado restringiu-se unicamente aos investimentos realizados pelo Ministério da Saúde. Nesse sentido, os resultados da avaliação não reproduzem e nem refletem, necessariamente, o quadro para todo o investimento de $\mathrm{P} \& \mathrm{D} / \mathrm{S}$ no período, pois ainda existem outros importantes atores no cenário público de financiamento destas pesquisas no país, como o Ministério da Ciência e Tecnologia (através de suas agências de fomento, CNPq e FINEP), as FAP, o setor privado, dentre outros. Ao mesmo tempo, é bastante razoável supor-se que a pretendida indução se dirija, primeiramente, 
aos recursos aplicados pelo próprio Ministério da Saúde, gestor da PNCTI/S e um dos principais financiadores da $\mathrm{P} \& \mathrm{D} / \mathrm{S}$, e que, caso esta já esteja se fazendo presente no período, então se possam observar seus primeiros efeitos.

A distribuição dos investimentos segundo os destinatários dos recursos não representa, necessariamente, os volumes definitivamente recebidos pelas categoriais institucionais em questão. Mesmo após tentativas exaustivas, não foi possível em certa proporção de casos identificar os receptores finais dos recursos, ficando estes atribuídos a possíveis intermediários no fluxo dos investimentos, como as FAP. Ainda que isso possa induzir certo grau de distorção no perfil de destinatários de recursos, a proporção é bastante pequena $(0,16 \%$ do total de recursos) e é fruto das limitações decorrentes da inexistência de sistemas de informação mais adequados ao mapeamento aqui proposto dos investimentos em $\mathrm{P} \& \mathrm{D} / \mathrm{S}$. Mencione-se também que, por vezes, o Ministério da Saúde repassa recursos para organismos internacionais - como OPAS e UNESCO - que, em parceria com instituições nacionais de pesquisa públicas e privadas realizam projetos de interesse desse Ministério 13. Quando não foi possível identificar esses usuários finais dos recursos, eles foram atribuídos aos organismos internacionais que funcionaram como intermediários nos fluxos $(2,4 \%)$.

Outra limitação é relativa à informação disponível à mensuração e categorização dos fluxos financeiros, que se desdobra em dois aspectos. $\mathrm{O}$ primeiro refere-se à disponibilidade e acesso à informação, que se apresentam de forma bastante heterogênea e diferenciada nos diversos agentes intraministeriais de financiamento. A relativa escassez de dados em forma informatizada dificulta e torna bastante trabalhosa e custosa (em termos de recursos humanos e tempo) o tipo de levantamento executado e obsta sua realização em uma base mais regular. Em alguns casos, está ocorrendo processo de migração para sistemas institucionais informatizados de coleta (por exemplo, na ANVISA e Fiocruz). Buscou-se, na execução da pesquisa, minorar essas dificuldades com as seguintes estratégias: (1) busca não apenas nas bases existentes, mas também pela solicitação direta de informações às instituições; (2) estabelecimento de diferentes formas de contatos prévios com estas; (3) geração de instrutivos de pesquisa, explicitando a caracterização do que constituía pesquisa em saúde e as classificações utilizadas; (4) disponibilização contínua de plantão telefônico para esclarecimentos; (5) cruzamento dos dados obtidos de financiadores e executores das $\mathrm{P} \& \mathrm{D} / \mathrm{S}$. Mesmo com todos estes esforços, a impossibilidade de se conhecer, a contento e de forma precisa, o real efetivo de pesquisas financiadas, seja no todo, seja em algum órgão específico, impede que se possa assegurar que não tenham ocorrido eventuais perdas ou distorções.

Em se desejando um acompanhamento evolutivo dos financiamentos em $\mathrm{P} \& \mathrm{D} / \mathrm{S}$, é importante que o Ministério da Saúde estimule internamente à sua estrutura, o registro adequado e desenvolvimento de sistemas de informação direcionados à monitorização destas atividades. Os primeiros passos nesse sentido foram realizados com a introdução recente da base gerencial de dados Pesquisa Saúde, que congrega informações sobre estudos financiados pelo DECIT. Seria fundamental, entretanto, que iniciativas semelhantes estivessem presentes em outros órgãos, de modo a contemplar o espectro mais amplo de todos os envolvidos nestes financiamentos.

Outro ponto a ponderar refere-se à qualidade, muitas vezes precária, da informação disponível para classificação, em certos casos, restrita apenas ao título da pesquisa e a poucas informações adicionais, insuficientes para permitir sua adequada categorização. Essa paucidade dificulta, particularmente, as classificações que se estendem para além do primeiro nível da ANPPS, restringindo a apreensão e possibilidade de análises mais detalhadas das temáticas ou áreas que estão realmente recebendo os investimentos e sua comparação com as prioridades estabelecidas dentro de cada subagenda.

Por ressaltarem-se ainda alguns aspectos inerentes à própria estrutura da ANPPS. O primeiro refere-se à possibilidade de uma dada pesquisa ser classificada em mais de uma subagenda. Em parte, tal é resultado da própria característica da pesquisa em saúde, permeada pela contribuição e participação de vários campos disciplinares e temáticos. Ademais, tem-se a forma particular de organização da ANPPS, com sua subdivisão em diversos níveis, conduzindo a graus variados de superposição entre as subagendas, por exemplo, quando pesquisas relacionadas ao desenvolvimento e testagem de tecnologias de rastreio para câncer de colo do útero são tratadas tanto na subagenda saúde da mulher, quanto na de doenças não-transmissíveis (neoplasias) e na de pesquisa clínica.

É importante mencionar que a classificação pelas subagendas deu-se em função do objeto da pesquisa. Nesse sentido, merece destaque o modelo de editais no período em tela, os quais, embora direcionados a subagendas específicas alimentação e nutrição - definem temas e linhas de apoio (por exemplo, desenvolvimento de metodologias de intervenção voltadas à educação nutricional de crianças e adolescentes) que, pela 
metodologia utilizada, determinou a computação desses recursos nas demais subagendas pertinentes.

Ao mesmo tempo, quando se examina a ANPPS, em particular os diversos subníveis, observa-se a existência de lacunas nos níveis de investigação dentro de cada uma das subagendas. Por exemplo, tomando por base a subagenda doenças transmissíveis, há um grande número de prioridades de pesquisa relacionadas à hanseníase, mas outras condições ainda relevantes no país, como o sarampo e o tétano, inexistem de forma explicitada ou são apenas marginais. Essas especificidades da ANPPS, por sua vez, são fruto do seu processo de elaboração, organizado em torno de diversas subcomissões independentes, com os naturais conflitos e disputas de poder subjacentes.

Nesse sentido, há que se salientar também que agendas de prioridades em pesquisa - sentido geral e não especificamente a nossa - são constructos dinâmicos, que devem ser reavaliados ao longo do tempo, para dar conta tanto de um processo progressivo de refinamento quanto das mudanças decorrentes do perfil sanitário, que se renova continuamente.

\section{Conclusões}

O trabalho envolveu o mapeamento dos dispêndios em P\&D/S do Ministério da Saúde no triênio 2003-2005 à luz da ANPPS. Não foi seu objetivo, embora se afirme como muito relevante, a discussão desse tipo de instrumento enquanto estratégia para superação das desigualdades em saúde ou de sua efetiva capacidade de aproximar as atividades de quem faz pesquisa daquelas de quem faz política.

Destaca-se o importante desafio à discussão das relações entre pesquisa em saúde e a sociedade, das estratégias de difusão e acesso ao conhecimento, dos mecanismos de financiamento e de controle social. Igualmente, entende-se que, mesmo garantindo-se a seletividade do fomento, isso não necessariamente significa que os resultados das pesquisas financiadas efetivamente permitam a redução do desequilíbrio entre necessidades sanitárias e a pesquisa em saúde.

Todas estas questões e outras mais carecem de maiores aprofundamentos, para que se construa um percurso para a consolidação de um cenário científico, que contribua eficazmente para o benefício da saúde e para o desenvolvimento mais generalizado.

\section{Resumo}

O trabalho mapeou a aplicação dos recursos financeiros em pesquisa e desenvolvimento em saúde $(P \& D / S)$ pelo Ministério da Saúde no período 20032005, conforme a Agenda Nacional de Prioridades de Pesquisa em Saúde (ANPPS), estabelecida em 2004. Utilizaram-se dados procedentes de pesquisa realizada com a finalidade primária de mensurar esses fluxos de investimento no período. Foi computado apenas o financiamento direto e efetivamente pago em pesquisas, excluindo-se dispêndios com salários. As pesquisas foram categorizadas segundo as 24 subagendas da ANPPS por dois pesquisadores independentes, com as discordâncias resolvidas por consenso. Foram aplica- dos cerca de $R \$$ 409,7 milhões, com uma concentração nas subagendas: doenças transmissíveis, complexo produtivo da saúde, pesquisa clínica, assistência farmacêutica e doenças não-transmissíveis (79\% do total). Todas as subagendas receberam algum financiamento no período. O estudo estabelece um marco zero para avaliações do potencial indutor deste instrumento e da aproximação entre os investimentos em $P \& D / S$ e as necessidades sanitárias.

Recursos Financeiros em Saúde; Agenda de Prioridades em Saúde; Política de Pesquisa em Saúde 


\section{Colaboradores}

R. M. Silva participou da concepção do estudo, análise e interpretação dos dados e redação do artigo. R. Caetano colaborou na concepção do estudo, revisão crítica e aprovação final da versão a ser publicada.

\section{Referências}

1. Morel CM. Neglected diseases: under-funded research and inadequate health interventions: can we change this reality? EMBO Rep 2003; 4 Spec No:S35-8.

2. Global Forum for Health Research. Monitoring financial flows for health research 2008: prioritizing research for health equity. Geneva: Global Forum for Health Research; 2008.

3. Global Forum for Health Research. 10/90 report on health research 2003-2004. Geneva: Global Forum for Health Research; 2004.

4. Guimarães R. Bases para uma política nacional de ciência, tecnologia e inovação em saúde. Ciênc Saúde Coletiva 2004; 9:375-87.

5. Departamento de Ciência e Tecnologia, Secretaria de Ciência e Tecnologia e Insumos Estratégicos, Ministério da Saúde. Da política à ação institucional: prioridades de pesquisa no Ministério da Saúde. Rev Saúde Pública 2006; 40:548-52.

6. Silva RM. Fluxos financeiros em pesquisa e desenvolvimento em saúde de 2003 a 2005: um exame dos investimentos do Ministério da Saúde segundo a Agenda Nacional de Prioridades de Pesquisa em Saúde [Dissertação de Mestrado]. Rio de Janeiro: Instituto de Medicina Social, Universidade do Estado do Rio de Janeiro; 2008.

7. Ministério da Saúde. Política Nacional de Ciência, Tecnologia e Inovação em Saúde. Brasília: Ministério da Saúde; 2006. (Série B. Textos Básicos em Saúde).

8. Ministério da Saúde. Agenda Nacional de Prioridades de Pesquisa em Saúde. Brasília: Ministério da Saúde; 2005. (Série B. Textos Básicos em Saúde).

\section{Agradecimentos}

Os autores agradecem aos pesquisadores Cid Manso de Mello Vianna, Mariana Miranda Autran Sampaio, Rodolfo Rego Deusdará Rodrigues, incluindo o apoio do digitador Artur Luiz Parreira Arêas que, juntamente com Rondineli Mendes da Silva e Rosângela Caetano, participaram da pesquisa original Fluxos de Recursos Financeiros para a Pesquisa e Desenvolvimento em Saúde no Brasil 2003-2005, cujos dados foram parcialmente utilizados no presente estudo.

9. Secretaria de Gestão Participativa, Ministério da Saúde. Brasília. Conferência Sérgio Arouca: relatório final. (Série D. Reuniões e Conferências).

10. Guimarães R, Santos LMP, Angulo-Tuesta A, Serruya SJ. Defining and implementing a National Policy for Science, Technology and Innovation in Health: lessons from the Brazilian experience. Cad Saúde Pública 2006; 22:1775-94.

11. Global Forum for Health Research. Monitoring Financial Flows for Health Research 2009: behind the global numbers. Geneva: Global Forum for Health Research; 2009.

12. Alano Jr. BP, Almario ES. Tracking country resource flows for health research and development (R\&D). Manila: Center for Economic Policy Research; 2000.

13. Ministério da Saúde. Fluxos de recursos financeiros para a pesquisa e desenvolvimento em saúde no Brasil: 2000-2002. Brasília: Ministério da Saúde; 2006. (Série C. Projetos, Programas e Relatórios).

14. Ministério da Saúde. Por que pesquisa em saúde? Brasília: Ministério da Saúde; 2007. (Série B. Textos Básicos de Saúde, Série Pesquisa para Saúde).

15. Caetano R, Vianna CMM, Sampaio MMA, Silva $\mathrm{RM}$, Rodrigues RRD. Uma análise dos investimentos do Ministério da Saúde do Brasil em pesquisa e desenvolvimento no período 2000-2002: uma linha de base para avaliações futuras a partir da implementação da Agenda Nacional de Prioridades em Pesquisa em Saúde. Ciênc Saúde Coletiva 2010; 15:2039-50

Recebido em 18/Ago/2009

Versão final reapresentada em 29/Nov/2010 Aprovado em 01/Fev/2011 